\title{
Intrahepatic Bile Duct Papillary Neoplasm with an Associated Invasive Carcinoma
}

National Cancer Institute

\section{Source}

National Cancer Institute. Intrahepatic Bile Duct Papillary Neoplasm with an Associated Invasive Carcinoma. NCI Thesaurus. Code C96949.

A papillary epithelial neoplasm that arises from the epithelium of the intrahepatic bile ducts and it is associated with an invasive carcinomatous component. The carcinomatous component is an intrahepatic cholangiocarcinoma. 